\title{
Cancer Care in the Era of COVID-19
}

Megan Tenet ${ }^{1}$

${ }^{1}$ Georgetown University School of Medicine

Keywords: cancer, coronavirus, covid-19, oncology

https://doi.org/10.52504/001c.13401

Georgetown Medical Review

Vol. 4, Issue 1, 2020

The increased vulnerability of patients with cancer to COVID-19 and the implementation of stay-at-home orders have made delivery of the normal standard of care challenging. Innovative practices and new guidelines have been developed by cancer centers worldwide to provide high-quality care while also decreasing the risk of infection with the novel coronavirus.

Patients with cancer are no strangers to hospitals. Their care is often multifactorial in nature and requires frequent trips for health care provider visits, chemotherapy, blood draws, hydration, radiation therapy, scans, biopsies, psychotherapy, and a myriad of other services and treatments. During the coronavirus disease 2019 (COVID-19) pandemic, cancer centers have had to significantly modify their treatment plans to keep patients at home and minimize the potential for them to become infected. These centers have faced a significant challenge in reconciling the need to maintain the same standard of cancer care while protecting their patients from a virus that could cause a devastating outcome.

Patients with cancer are particularly vulnerable to the novel coronavirus for a number of reasons. First, many patients with cancer are immunosuppressed. One source of immunosuppression is the disease itself because cancer cells are known to secrete immunosuppressive factors such as TGF- $\beta$, IL-10, and other cytokines that inhibit antitumor activity and weaken the immune system. ${ }^{1} \mathrm{~A}$ second source includes therapies, such as chemotherapy, radiation therapy, or bone marrow transplantation, in which systemic immunosuppression can be an unwanted adverse effect. The inability of the body to produce a robust immune response makes infectivity with COVID-19 particularly dangerous. Cancer is also more prevalent in older populations that are more likely to have the chronic comorbid conditions that are known to increase susceptibility to COVID-19 such as heart disease, hypertension, and chronic obstructive pulmonary disease. ${ }^{2}$ A retrospective study of patients with COVID-19 in China showed that those with cancer had a higher risk of severe events, such as requirement for ventilation or death, than patients without cancer. ${ }^{2}$ It was also observed that those with cancer decompensated at a faster rate than patients without cancer. ${ }^{3}$

Many routine practices for patients with cancer now pose significant risk due to the pandemic. Normally, these patients may visit their cancer center several times a month where they face crowded waiting rooms and interact with 
various staff members. Some travel a long distance for their care and use public transportation. They also are likely to attend visits with multiple loved ones for support due to the emotional burden of their disease. Given the increased vulnerability of this patient population and the Centers for Disease Control and Prevention recommendation to practice social distancing and avoid crowded spaces, new methods and guidelines have been implemented to keep patients safe.

Telemedicine has allowed health care providers to significantly decrease patient volume in their facilities. At Lombardi Cancer Center at MedStar Georgetown University Hospital, use of telemedicine has reduced the number of in-person visits occurring each day (Kristin Ferguson, DNP, RN, OCN, clinical operations manager at the Lombardi Cancer Center at Medstar Georgetown University Hospital; oral communication; May 2020). "Our providers are continuing to see patients in-person who cannot delay care, for example, those on active treatments such as chemotherapy. Even during a pandemic, cancer still needs to be treated in a timely, evidence-based manner. Most of our providers are also performing telemedicine visits with patients who do not need to come into the hospital. This has allowed us to prevent patients who are perhaps immunocompromised from leaving their house if they do not need direct care that day," Additionally, Ferguson said, chairs in clinic waiting rooms have been rearranged to allow those who do need to come in to remain at a safe distance from others. It is also not permitted to bring more than 1 other person to a physician visit, and no other persons are allowed in the chemotherapy suite with the patient. ${ }^{4}$

For cancer centers worldwide, the ability to communicate via telehealth has been an invaluable asset since stay-at-home orders were implemented. Telemedicine existed prior to the outbreak of COVID-19 but its use was relatively infrequent. In 2017, according to the Government Accountability Office, only about $1 \%$ of Medicare beneficiaries used telemedicine, and most resided in rural areas. ${ }^{5}$ A 2019 survey conducted by PricewaterhouseCoopers found that chief executive officers of $38 \%$ of US health care systems stated that there was no digital component whatsoever to their system at that time. ${ }^{6}$ Before the pandemic, regulations imposed by Medicare limited the use of telemedicine largely to patients in rural areas engaging in a virtual follow-up visit with a health care provider they had previously seen in person. ${ }^{5}$ However, in mid-March, as lockdowns across the nation began, the Trump administration waived various federal regulations to make telemedicine visits reimbursable by Medicare and Medicaid and accessible to patients nationwide. ${ }^{7}$ Following this announcement, the Department of Health and Human Services announced it would relax Health Insurance Portability and Accountability Act laws during this time and health care providers would not be penalized if they conducted telemedicine visits via FaceTime or Skype, which are not compliant with privacy law. "The advantages of telemedicine for oncology patients are that they do not have to leave their home and risk 
getting an infection and, therefore, they feel less anxious while they can still continue to receive the same care and advice from their medical oncologist during a telemedicine visit," Ferguson explained. ${ }^{4}$

While telemedicine has been a tremendous advancement, it is also important to acknowledge its limits. In a survey conducted by NORC at the University of Chicago, approximately half of patients who participated in telemedicine reported that their visit was equivalent to an in-person visit, but $40 \%$ were disappointed in the quality of their care. ${ }^{5}$ The accelerated launch of telemedicine into current practice has made proper training of health care providers challenging. Some physicians have warned of the danger of relying too much on telemedicine, and highlight the importance of a thorough physical examination. Many patients also have less sophisticated internet capacity, making blurry image quality, lags, and freezing of calls an unfortunate reality. These shortcomings are actively being addressed by health systems to improve telehealth as COVID-19 will continue to be a concern in the coming months and, possibly, years. ${ }^{8}$

For patients receiving active treatment, societies of clinical oncology worldwide, such as the American Society of Clinical Oncology and the European Society for Medical Oncology, have developed new guidelines. These guidelines include assigning low, medium, or high priority to patients using the Ontario Health/Cancer Care Ontario criteria. High-priority patients include those who are clinically unstable or have a condition that is life threatening, while low-priority patients are those who are stable enough to withstand a delay in treatment ${ }^{9}$ By stratifying patients in this way, cancer centers have been able to decrease volume in treatment areas, such as chemotherapy centers, making appropriate social distancing a more attainable goal. ${ }^{9}$ Information provided by societies of clinical oncology has been posted to their respective websites and frequently updated so that health care providers have easy access to the most up-to-date literature and recommendations.

Continuing radiation therapy during the COVID-19 crisis is particularly challenging due to its demanding schedule. Radiation therapy typically requires patients to visit their cancer center daily for a number of weeks. The American Society for Radiation Oncology has recommended that hypofractionated schedules, or dividing the total amount of radiation into larger doses and given over a shorter time, be used whenever possible. For patients receiving radiation for palliative purposes, it is advised that health care providers consider delaying treatment if it is considered safe for the patient. ${ }^{9}$

Surgeries and stem cell transplants have also been reconsidered for many patients. Across all medical specialties, it has been recommended that elective surgeries be delayed or canceled due to the shortage of intensive care unit beds and ventilators. Admitting patients for surgery also increases their chance of contracting COVID-19 in the hospital setting. Many oncology surgeries have been delayed at the discretion of the physician and the surgeon, depending 
on the status of the patient. ${ }^{9}$ A viable alternative to surgery for patients with early-stage disease is beginning their treatment with neoadjuvant therapy, such as chemotherapy or immunotherapy, followed by surgery at a later time. Hematopoietic stem cell transplantation is a common treatment for patients with liquid cancers such as leukemia, lymphoma, and multiple myeloma. It has been recommended that these transplants be delayed if possible to keep patients out of the hospital as well as to prevent the extreme immunosuppression caused by this procedure, leaving them even more susceptible to the harmful effects of COVID-19. ${ }^{9}$

Continuing clinical trials for new cancer drugs has been difficult. Trials of cancer drugs often offer patients who have exhausted standard therapies a chance to try a novel treatment. Unfortunately, at the urging of the Food and Drug Administration, many clinical trials have paused enrollment of new patients, and study protocols for existing patients currently in trials have been significantly modified to reduce patient visits. ${ }^{10}$ Appointments have been conducted by telemedicine whenever possible, and additional blood draws and biopsies for research purposes are being deferred. ${ }^{11}$ Many companies sponsoring trials have opted to ship experimental medicines directly to patients rather than have them pick up their drugs at a physician visit. ${ }^{10}$ In the midst of major disruptions to data collection, sponsoring companies have urged investigators to prioritize adhering to the study scan schedule as much as possible in order to have some indication of whether the drug has potential for success. For novel therapies, radiographic evidence of reduction in tumor size is often the best way to measure efficacy. Investigators certainly understand the importance of this data collection to advancing research, but they also recognize that their primary responsibility is to protect their patients' safety, so this may not always be possible. Because infusion therapy is limited to administration in a chemotherapy center, many patients taking experimental infusion therapies who traveled long distances for a particular trial have unfortunately had to withdraw because of the risks posed by traveling. Studies of experimental therapies, such as CAR-T cell treatments, have been halted altogether because of the danger of significant adverse effects that could potentially require intensive care. ${ }^{10}$ In the long-term, the pandemic will have had a considerable detrimental effect on cancer research due both to decreased enrollment and a loss of data from specimen collection and assessments while stay-at-home orders are in place.

Ultimately, providing quality cancer care while keeping patients safe relies on good clinical judgment by health care providers. Physicians must assess the riskbenefit ratio of every situation of a patient's care. As with all things in medicine, no two patients are alike, and the disease and social situation of every patient with cancer pose a unique set of challenges that must be considered when deciding the most appropriate path forward during this unprecedented time. 
"Each patient and their case are individual and need to be treated as such and our providers are excellent at tailoring their care to meet patient needs" said Ferguson.

Lastly, the mental and emotional impact on patients with cancer during this crisis cannot be overstated. Depression, anxiety, and other mental health disorders are common comorbid conditions for those with cancer and are increasingly challenging to manage during this period of stress and uncertainty. ${ }^{12}$ Because of their increased susceptibility to COVID-19, many patients with cancer have been advised to avoid contact with others as much as possible, leaving them isolated from family and friends who often are sources of support. It is imperative that health care providers be cognizant of their patients' mental and emotional state, and provide the resources that patients need to maintain wellness during the pandemic. Ferguson stressed that in order to best provide this compassionate care, health care providers must remember to make time for self-care. "I think understanding the stress that many are under during this unprecedented time is key to helping others cope. The best thing medical professionals can do is ensure they themselves are remaining healthy and caring for themselves physically, emotionally, and mentally to remain resilient in caring for our patients." 4 


\section{REFERENCES}

1. Chambers W, Rabinowich H, Herberman RB, et al. Mechanisms of immunosuppression. In: Kufe DW, Pollock RE, Weichselbaum RR, eds. Holland-Frei Cancer Medicine. Vol 6. 6th ed. BC Decker; 2003.

2. Yang J, Zheng Y, Gou X, et al. Prevalence of comorbidities and its effects in patients infected with SARS-CoV-2: A systematic review and meta-analysis. International Journal of Infectious Diseases. 2020;94:91-95. doi:10.1016/j.ijid.2020.03.017

3. Liang W, Guan W, Chen R, et al. Cancer patients in SARS-CoV-2 infection: A nationwide analysis in China. The Lancet Oncology. 2020;21(3):335-337. doi:10.1016/s1470-2045(20)30096-6

4. Dai M, Liu D, Liu M, et al. Patients with cancer appear more vulnerable to SARS-COV-2: A multi-center study during the COVID-19 outbreak. Cancer Discov. 2020;10(6):783-791. doi:10.1158/2159-8290.cd-20-0422

5. Keesara S, Jonas A, Schulman K. Covid-19 and health care's digital revolution. N Engl J Med. 2020;382(23):e82. doi:10.1056/nejmp2005835

6. Span P. With red tape lifted, Dr Zoom will see you now. The New York Times. https://www.nytimes.com/2020/05/08/health/coronavirus-telemedicine-seniors.html. Published May 8, 2020. Accessed May 13, 2020.

7. Webster P. Virtual health care in the era of COVID-19. The Lancet. 2020;395(10231):1180-1181. doi:10.1016/s0140-6736(20)30818-7

8. Mehrotra A, Ray K, Brockmeyer DM, Barnett ML, Bender JA. Rapidly converting to "virtual practices": Outpatient care in the era of Covid-19. NEJM Catalyst .2020;1(2). doi:10.1056/ CAT.20.0091

9. Gosain R, Abdou Y, Singh A, Rana N, Puzanov I, Ernstoff MS. COVID-19 and cancer: A comprehensive review. Curr Oncol Rep. 2020;22(5):53. doi:10.1007/s11912-020-00934-7

10. Joseph A. Patients, drug makers grapple with how to continue cancer trials during the coronavirus. STAT News. https://www.statnews.com/2020/04/29/patients-drug-companiesgrapple-cancer-trials-coronavirus/. Published April 29, 2020. Accessed May 16, 2020.

11. US Food and Drug Administration. FDA Guidance on Conduct of Clinical Trials of Medical Products during COVID-19 Public Health Emergency: Guidance for Industry, Investigators, and Institutional Review Boards.; 2020. https://www.fda.gov/regulatory-information/search-fdaguidance-documents/fda-guidance-conduct-clinical-trials-medical-products-duringcovid-19-public-health-emergency. Accessed May 14, 2020.

12. Walker J, Holm Hansen C, Martin P, et al. Prevalence of depression in adults with cancer: A systematic review. Annals of Oncology. 2013;24(4):895-900. doi:10.1093/annonc/mds575 